\title{
Enhanced Human Identity and Gender Recognition from Gait Sequences using SVM and MDA
}

\author{
Alka Saini \\ Department of Electronics and Communication \\ Chandigarh University \\ Gharuan, Mohali, Punjab, INDIA 140413
}

\author{
Harpreet Singh \\ Department of Electronics and Communication \\ Chandigarh University \\ Gharuan, Mohali, Punjab, INDIA 140413
}

\begin{abstract}
The identification through biometric is a better way because it associate with individual not with information passing from one place to another. There are numerous biometric measures which can be used to help derive an individual identity. It is the biometric process and has many advantages over other biometric traits such as face, iris, fingerprint, palm print, etc. Most current approaches make the unrealistic assumption that persons walk along a fixed direction or a pre-defined path. Gait is the manner or style of moving on foot. Human Gait recognition identifies the individuals by the way in which they walk. Recognition of an individual is an important task to identify people. A gait sequence is collected from arbitrary walking directions. In this paper we present the approach of human identity and gender recognition using Model based features extraction and SURF for matching along with SVM and MDA algorithm.
\end{abstract}

Keywords- Gait recognition, biometrics, arbitrary direction, SVM, MDA.

\section{INTRODUCTION}

Biometrics was about perceiving individuals naturally. Basically we determine a set of numbers that are interesting to a specific individual. The biometrics began long back, with chip away at programmed face recognition. The shot were taken at utilizing human depiction for recognition, which was associated with relating workstation vision properties with those of human vision [1]. Authentication is a region which has developed throughout the most recent decades, and will keep on growing later on. It is utilized within numerous places today and being verified has turned into a day by day propensity for generally individuals.

Biometric systems are highly used to avoid the unauthorized access. Human Gait recognition is the technique in which we identify the individuals by the way they walk. In early 1990's Niyogi and Adelson first attempted the human recognition using Gait [2]. Gait based recognition is more suitable in video surveillance applications because of following advantages: Unobtrusive, Distance Recognition, Reduced detail and Difficult to conceal. The traditional frameworks have a few issues as far as comfort and execution. This can result in much hinder for the client and it is additionally conceivable to take dormant data from the finger impression sensor. Also, the state of the finger surface (e.g. sweat, dryness) and skin bending can result in debased recognition exactness. For face recognition, execution exceedingly relies on upon facial outflows and brightening, which can change. Iris recognition is most dependable regarding exactness, yet the catching gadget is costly and might be awkward contrasted with other biometric frameworks. "A physiological or behavioral trademark is which could be utilized to recognize and check the personality of a single person" [3]. Gait analysis has also been applicable in our daily life. It is applicable in smart surveillance systems, in virtual reality, in advanced user interfaces and in motion analysis. It has been pulled in enthusiasm as a technique for ID on the grounds that it is not obtrusive and does not oblige the subject's participation. Gait recognition innovation is not constrained to security applications -analysts additionally imagine restorative applications for the engineering. For instance, perceiving changes in strolling examples at an opportune time can help to distinguish conditions, for example, Parkinson's infection and numerous sclerosis in their soonest stage. Gait recognition could be utilized from a separation that making it appropriate to recognizing the culprits at a wrongdoing scene [4]. The fig.1 shown below, shows the propose gait recognition system.

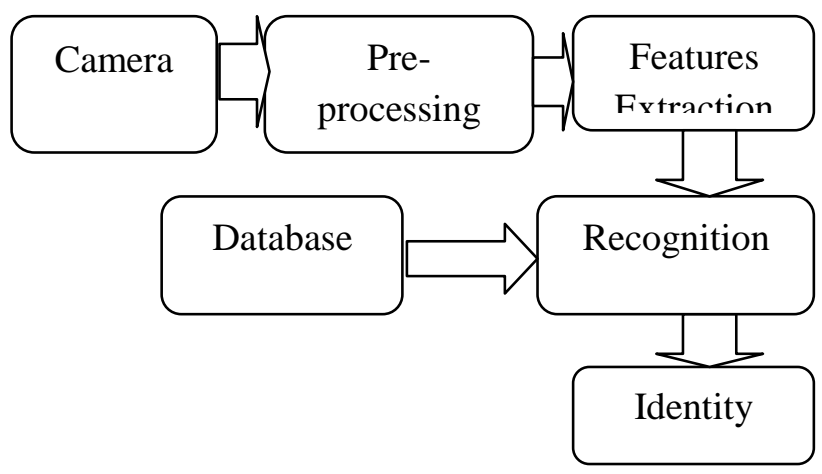

Fig. 1 Gait Recognition System

This illustrates how the capture video is converted into the frames and after that background subtraction is applied on that so as to remove the distortion or noise present in the image. Background subtraction is a process in which the object in a particular location is extracted [5]. Each of the frames in the image sequence is subtracted from a background model of the respective image sequence. After that features are extracted by using different model based approaches eg. hanavan's model, by using this model various features can be extracted. The individual is recognized by comparing the obtained characteristics or features with the ones previously stored in the database. This paper presents the review of the methods proposed to identify human and gender recognition.

\section{METHODOLOGY}

In proposed system we used SVM and MDA methods for classification. We have to process the video into frames, after the frame conversion we have find the object in this video by 
using the object have to apply the AP clustering for feature extraction. By using SVM classifier to classify the videos and finding the gender. Compare to existing system our method is giving better output. Here, the methodology of the proposed work for the gait recognition system is explained. Firstly the phases of the gait recognition system are explained and then the algorithms used in the process are explained. The first phase is the load video in which the system will load a random video which is selected, as shown in the fig below there is a button with the display of load video by clicking on the button it goes to the browse window from where we choose their video destination and then the video is loaded into the system. The proposed methodology shown in fig. 2 is divided into following steps:

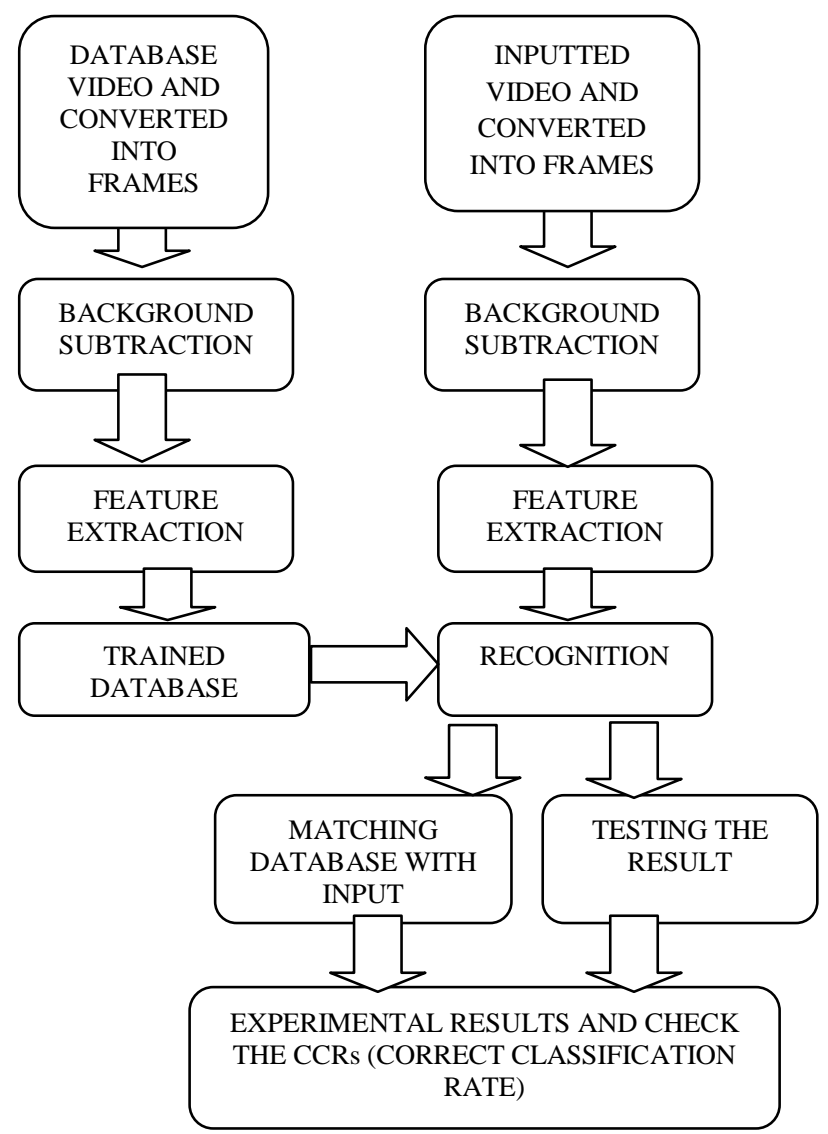

Fig. 2 Proposed Methodology Flowchart

\section{RELATED WORKS}

Jiwen Lu, Gang Wang and Pierre Moulin worked on the human identity and gender recognition from gait sequences with arbitrary walking directions. It has a proposed method based on Sparse Reconstruction based Metric Learning (SRML). The authors in [6] investigate the problem of human identity and gender recognition from gait sequences with arbitrary walking directions. Most datasets assume that people walk along a fixed direction or a pre-defined path but this is unrealistic because people walk freely and the walking direction may be time varying. To study this new problem a new gait database named ADSC-AWD (Advanced Digital Sciences Centre-Arbitrary Walking Directions) is constructed in which people walk freely in the scene, and the walking directions are arbitrary and time-varying. Cluster-based averaged gait image(C-AGI) is computed as the feature. A method is proposed, SRML (Sparse Reconstruction Based
Metric Learning) for discriminative gait feature extraction. The other work related to human identification is done by M. Jeevan, Neha Jain, M. Hanmandlu and Girija Chetty [7]. In this Pal and Pal Entropy is proposed to achieve reasonable results on a large database like CASIA dataset B and C. Maodi Hu, Yunhong Wang, Zhaoxiang Zhang and De Zhang [8] proposes a modeling approach for gait-based gender classification which includes shape features extraction. CASIA and IRIP gait database are used for gender recognition. The conditional random field (CRF) is used for features extraction. X. Huang and N. V. Boulgouris in "Gait recognition with shifted energy image and structural features extraction". By S. Yu, T. Tan, K. Huang, K. Jia, and X. Wu. "A study on gait-based gender classification". By X. Li, S. Maybank, S. Yan, D. Tao, and D. Xu. in "Gait components and their application to gender recognition" and by Qiong Cheng, Bo Fu and Hui Chen, "Gait Recognition Based on PCA and LDA".

\section{PROPOSED APPROACH}

\subsection{Video Capture}

The first phase of the gait recognition system includes the input video where a video is taken as a input, as shown in figure 3 and the video is converted into n number of frames. Method of accurate tracking person in indoor/outdoor surveillance video stream obtained from static camera. Example: a camera on the front door or anywhere in complex which can store gait sequences of a moving person on foot, so that video can be used for further processing.

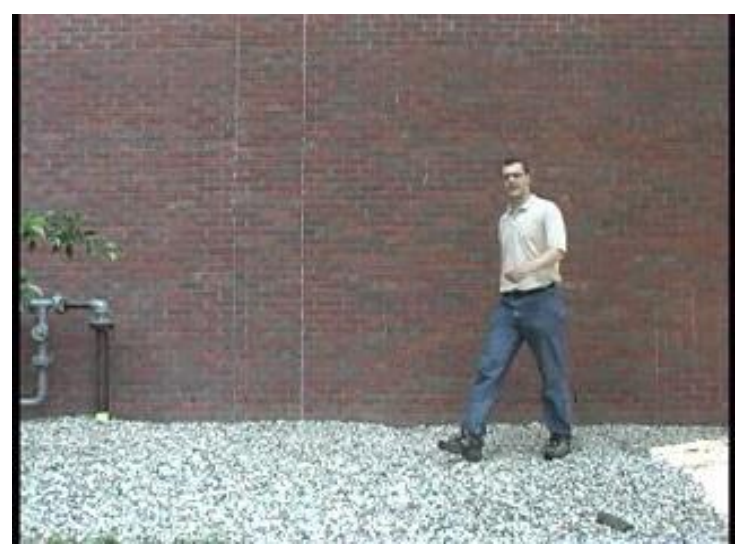

Fig. 3: Input video

\subsection{Background Subtraction}

The background subtraction method is the common method of motion detection. It is a technique that uses the difference of the current image and the background image to detect the motion region. Its calculation is simple and easy to implement. Background subtraction is used to subtract the moving objects and to obtain the silhouette [9]. Background subtraction is a process in which binary images are generated containing black and white (moving pixels) also known as binary silhouettes. Silhouette is defined as a region of pixels of the walking person [10]. In figure 4 subtraction of background is shown. To implement the background subtraction the main motive is to make the image more smoothing and useless part will be removed. There are many challenges in developing a good background subtraction algorithm. First, it must be robust against changes in illumination. Second, it should avoid detecting non-stationary background objects such as moving leaves, rain, snow, and shadows cast by moving objects. 


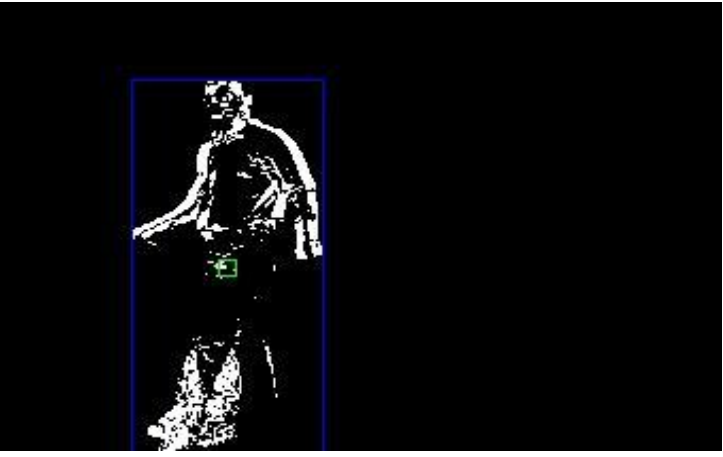

Fig. 4: Background subtraction

So for this, we use two different things Equation and Median filter.

\subsection{Feature Extraction}

Feature extraction is based upon the silhouette analysis. Feature selection is an essential venture in gait recognition. The peculiarity must be powerful to working conditions and ought to yield great separate capability crosswise over people. Every gait arrangement is separated into cycles. Hanavan's model is used for feature extraction for human identification and some of the important feature which was not used till date has been used in this system. Hanavan's model is the latest and fastest model based approaches for feature extraction. Fig. 5 shows the hanavan's model.

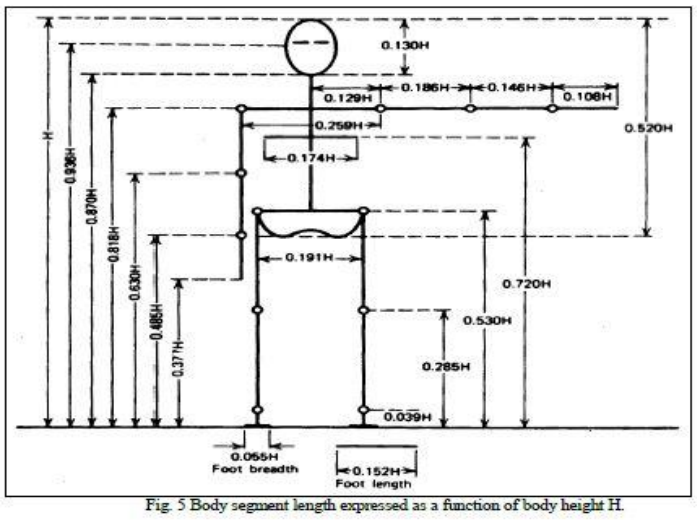

Fig.5 Hanavan's model

On the basis of above hanavan's model processing is done on the input data frames taking length of the parameters calculations are performed. As shown in the figure length of each part of body is given a parameter size on the basis of which calculations are performed. For gender recognition we use C-AGI as gait features. Given a gait sequence, assume there are Nk frames in the kth cluster, we then compute the CAGI as the gait feature:

$$
G k(x, y)=\frac{1}{N k} \sum_{p=1}^{N k} \operatorname{Ipk}(x, y)
$$

where $\operatorname{Ipk}(\mathrm{x}, \mathrm{y})$ is the pth human silhouette in the kth cluster, and $\mathrm{x}$ and $\mathrm{y}$ are 2-D image coordinates. C-AGI is the average silhouette of all frames within the same cluster.

\subsection{Recognition}

Recognition is the final step of gait-based person identification. In this step input test video sequences are compared with the trained sequence in the database. Recognition is done using support vector machine (SVM) and Multi linear discriminant analysis (MDA) classifier. These are discussed below.

\subsubsection{Support Vector Machine (SVM)}

The Support Vector Machine (SVM) is a method which was introduced in 1992 by Boser, Guyon, and Vapnik. The SVM classifier is generally utilized as a part of bioinformatics (and different orders) because of its profoundly exact, ready to figure and procedure the high-dimensional information, for example, gene interpretation, and excitability in displaying assorted wellsprings of information. The SVM classifier is widely used in bioinformatics and other field because it is very accurate, can process and calculate the high-dimensional data such as gene expression.

\section{Algorithm 1: Simple SVM}

Step 1: Candidate SV $=$ \{closest pair from opposite classes $\}$

Step 2: while there are violating points do

Find a violator

Step 3: candidateSV $=$ candidateSV $^{\mathrm{U}}$ violator

if any $\alpha_{p}<0$ due to addition of $c$ to $S$ then

Step 4: candidateSV $=$ candidateSV $\backslash p$

repeat till all such points are pruned

Step 5: end if

Step 6: end while

SVM is robust and thus strong generalization ability. It proved itself to have better performance on test data in both practice and theory.

\subsubsection{Multi Linear Discriminant Analysis (MDA)}

MDA is a technique, in which we can identify three or more class classifications. The main aim of this technique is to minimize difference between each class and maximize distance between different classes[11]. We summarize the advantages of Multilinear Discriminant Analysis (MDA), [12] as follows: MDA helps to solve the small sample size problem. The available feature dimension of LDA is theoretically limited by the number of classes in the data, whereas the MDA is not and the computational cost can be reduced to a large extent.

\subsection{Matching}

There are some important steps in features-based image matching method. First, features of image are extracted, second, matching between the features, third, completing the features matching in the different images. Moving target characterization calculation is utilized separate person (i.e., passerby) from other closer view objects (viz., vehicles). Surf feature is used in this system for matching. Surf feature is used for matching in this system it is a robust local feature detector that can be used in computer vision tasks like object recognition. SURF adds a lot of features to improve the speed 
in every step. Analysis shows it is 3 times faster than SIFT while performance is comparable to SIFT. SURF is good at handling images with blurring and rotation, but not good at handling viewpoint change and illumination change. It approximates or even outperforms previously proposed schemes with respect to repeatability, distinctiveness, and robustness, yet can be computed and compared much faster.

\section{EXPERIMENTAL RESULTS}

In this section, we perform gait based human identity and gender recognition experiments to verify the efficacy of our proposed approach. The comparison of the accuracy is done for every method is one with the given values to the proposed work. The accuracy rate is much higher in the proposed work as compare to the previous working methods.

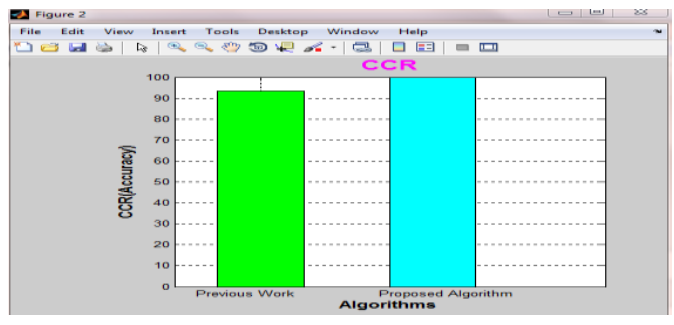

Fig 6: Graph of comparison of pervious work and proposed work

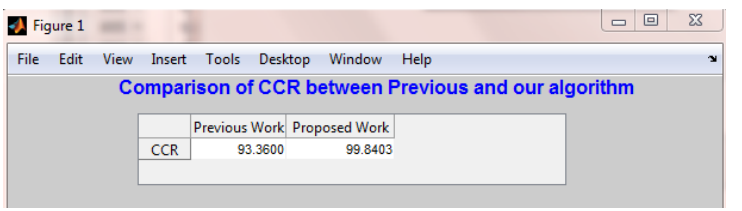

Fig 7: Comparison of CCR between previous and proposed algorithm

The above graph shows the comparison of the CCR values so as shown in the graph CCR Value of proposed system is almost $99 \%$.

\section{CONCLUSION AND FUTURE SCOPE}

This paper presents that enhanced human identification and gender recognition from gait sequences using SVM and MDA. A large number of experimental results have demonstrated the validity of the proposed algorithm. Although accomplished under some simplified assumptions like previous work, this work has been proven to be an encouraging progress to gait-based human identification.

Future work involves Gait based recognition has been portrayed in connection of individual validation Execution rate must be upgraded for obstacle, attire style conditions and moreover for unique walking examinations.

Even though we are getting promising results with the proposed approach, it has to be improved for large data bases. Performance rate have to be improved for occlusion, clothing style conditions and also for different walking considerations.

\section{REFERENCES}

[1] A. Elgammal, D. Harwood, L. S. Davis, "Nonparametric background model for background subtraction", Proc. 6th European Computer Vision, Vol. 2, May 2000, pp. 751-767

[2] S. Niyogi and E. Adelson, "Analyzing Gait with Spatiotemporal Surfaces," Proc. IEEE Workshop NonRigid Motion, pp. 24-29, 1994.
[3] A. Veeraraghavan, A. Chowdhury, and R. Chellappa, "Role of shape and kinematics in human movement analysis", In CVPR, pages 730-737, June 2004.

[4] J. Han and B. Bhanu, "Individual recognition using Gait energy image", IEEE Transactions on Pattern Analysis and Machine Intelligence, 2006.

[5] Lili Liu, Yilong Yin, Wei Qin, Ying Li, "Gait Recognition based on Outermost Contour ," International Journal of Computational Intelligence Systems, Vol. 4, September 2011, pp. 1090-1099

[6] Jiwen Lu, Gang Wang and Pierre Moulin. "Human Identity and Gender Recognition from Gait Sequences with Arbitrary Walking Directions". IEEE Transactions on Image Processing, 2013.

[7] M. Jeevan, Neha Jain, M. Hanmandlu and Girija Chetty. "Gait Recognition Based on Gait Pal and Pal Entropy Image”. IEEE International Conference, 2013.

[8] Maodi Hu, Yunhong Wang, Zhaoxiang Zhang and De Zhang. "Gait-Based Gender Classification Using Mixed Conditional Random Field". IEEE Transactions on Systems, Mans and Cybernetics, Part B: Cybernetics, 2011.

[9] M.Pushparani, D.Sasikala, "A Survey of Gait Recognition approach using PCA \& ICA", Global Journal of Computer Science and Technology Network, Web \&Security, Vol. 12, Issue 10, version 1.0, May2012.

[10] A.Hayder, J.Dargham, A.Chekima, G.M.Ervin,, "Person Identification Using Gait", International Journal of Computer and Electrical Engineering, Vol. 3, No. 4, August 2011.

[11] R.O. Duda, P.E. Hart, D.G. Strok, "Pattern Classification", Second Edition, Wiley,2000.

[12] Shuicheng Yan, Dong Xu, Qiang Yang, Lei Zhang, Xiaoou Tang and Hong-Jiang Zhang, "Multi-linear Discriminant Analysis for recognition ", IEEE Transactions on Image Processing, Vol. 16, no.1, January 2007.

[13] Dong Hui and Han Dian Yuan. "Research of Image Matching Algorithm Based on SURF Features". IEEE International Conference on Computer Science and Information Processing, 2012

[14] X. Huang and N. V. Boulgouris. "Gait recognition with shifted energy image and structural features extraction". IEEE Transactions on Image Processing, 21(4):22562268, 2012

[15] S. Yu, T. Tan, K. Huang, K. Jia, and X. Wu. "A study on gait-based gender classification". IEEE Transactions on Image Processing, 18(8):1905-1910, 2009.

[16] X. Li, S. Maybank, S. Yan, D. Tao, and D. Xu. "Gait components and their application to gender recognition". IEEE Transactions on Systems, Mans, and Cybernetics, Part C: Applications and Reviews, 38(2):145-155, 2008.

[17] Qiong Cheng, Bo Fu and Hui Chen, "Gait Recognition Based on PCA and LDA". International Computer Science and Computational Technology, pp. 124-127, Dec. 2009. 
[18] Pal, N.R., Pal, S.K., "Entropy: A new definition and its applications", IEEE Trans. Systems Man Cybernet. SMC-21, 1260-1270.

[19] Y. Chai, Q. Wang, R. Zhao, and C. Wu, "A new automatic Gait recognition method based on the perceptual curve", IEEE International Conference on Intelligent Information Communication Technologies for Better Human Life, 2005.

[20] R. Tanawongsuwan and A. Bobick, "Gait recognition from time normalized joint-angle trajectories in the walking plane", In Proceedings of IEEE Computer Society Conference on Computer Vision and Pattern Recognition, 2001.

[21] R. Collins, R. Gross, and J. Shi. "Silhouette-based human identification from body shape and Gait", In Proceedings of International Conference on Automatic face and Gesture Recognition, U.S.A 2002.

[22] Konstatinus, Huang Xinhan, Peng Gang, "Simplified SIFT Feature Point Detecting Method", AROC, 25(7): 2213-2215, 2008.
[23] J. Han and B. Bhanu, "Individual recognition using Gait energy image", IEEE Transactions on Pattern Analysis and Machine Intelligence, 2006.

[24] Ai-Hua, Wang Ji, Wei Liu, "Gait Recognition Method Based on positioning human body joints" International Conference on wavelet analysis and pattern recognition, Nov 2007.

[25] Y. Chai, Q. Wang, R. Zhao, and C. Wu, "A new automatic Gait recognition method based on the perceptual curve", IEEE International Conference on Intelligent Information Communication Technologies for Better Human Life, 2005.

[26] R. Collins, R. Gross, and J. Shi. "Silhouette-based human identification from body shape and Gait", In Proceedings of International Conference on Automatic face and Gesture Recognition, U.S.A 2002.

[27] Shuming Jiang, Yufei Wang, Yuanyuan Zhang and Jiande Sun, "Real Time Gait Recognition System based on Kinect Skeleton Feature", IEEE Computer Society Conference on Computer Vision and Pattern Recognition, Jan 2015 